\title{
Hydrodynamique tidale du lagon de Mayotte : observations in-situ et modélisation
}

\author{
Déborah Idier ${ }^{1}$, Emmanuel Romieu ${ }^{1}$, Matthieu Delattre ${ }^{1}$, Rodrigo \\ Pedreros $^{1}$ et Ywenn De La Torre ${ }^{2}$
}

${ }^{1}$ BRGM, Service Aménagement et Risques Naturels, 3 av. C. Guillemin, BP 6009, 45060 Orléans Cedex 2

d.idier@brgm.fr, e.romieu@brgm.fr,.m.delattre@brgm.fr, r.pedreros@brgm.fr

${ }^{2}$ BRGM, Service Géologique de La Réunion, 5 rue Ste Anne BP906, 97478 Saint Denis Cedex

y.delatorre@brgm.fr

\section{Résumé :}

L'hydrodynamique du lagon de Mayotte est étudiée par observations in-situ et modélisation. Huit capteurs de pression et vitesse ont été mouillés pendant un mois à l'automne 2007, autour de l'île principale et dans les passes. Ces mesures sont traitées pour en extraire les composantes de marée en niveau d'eau et courants. Un modèle hydrodynamique couvrant tout le lagon a été mis en place. Les mesures et résultats du modèle permettent ainsi d'obtenir des informations quantitatives sur la marée à l'échelle du lagon de Mayotte. En particulier, ils mettent en évidence : (1) une variabilité spatiale des composantes de marée au sein du lagon, (2) une variabilité spatiale des courants de marée, atteignant $1 \mathrm{~m} / \mathrm{s}$ dans les passes.

\begin{abstract}
:
This paper investigates the hydrodynamics in the Mayotte lagoon. For that purpose, eight pressure and current sensors were deployed on the lagoon sea bed, around the main island and in the coral reef inlets, during one month, in November-December 2007. The data were processed in order to extract and to characterise the tidal components of the water level and current. A 2DH hydrodynamic model is set up. The measurement and model results give a picture of the tidal hydrodynamics at the lagoon scale. They put forward: (1) the spatial variability of the tidal components, (2) the spatial variability of tidal currents, reaching $1 \mathrm{~m} / \mathrm{s}$ in the inlets.
\end{abstract}

Mots-clés :

Mayotte - lagon - courants - marée - modélisation - code MARS. 


\section{$1 \quad$ Introduction}

Avec une surface d'environ $1500 \mathrm{~km}^{2}$, le lagon de Mayotte (Figure 1), situé dans l'Océan Indien au Nord Ouest de Madagascar, est un des plus grands lagons au monde. Mayotte connait à l'heure actuelle une explosion démographique $(+5,7 \%)$ telle que la pression sur le milieu naturel est de plus en plus intense. Dans un tel environnement, les pouvoirs publics s'efforcent d'avoir une gestion cohérente des masses d'eau de l'île, notamment sur les problématiques de rejets des eaux usées dans le lagon (GOURBESVILLE et THOMASSIN, 2000). Cette gestion cohérente passe par une connaissance de la courantologie à l'échelle du lagon.

Jusqu'à présent, des travaux de modélisation des courants ont été effectués, soit à l'échelle de Madagascar et ses environs sur les grands courants océaniques, soit à l'échelle d'une portion du lagon (ne dépassant pas le $5^{\text {ième }}$ de la surface du lagon) sur les courants de marée et/ou induits par le vent. Ainsi, on peut noter les travaux de modélisation 2DH de GOURBESVILLE et THOMASSIN (2000) et du SIEAM (2005) entre l'île principale et Petite Terre (Figure 1b).

Le but de ce travail est, à partir de mesures hydrodynamiques et de travaux de modélisation, de mieux connaître la courantologie à l'échelle de tout le lagon. Dans le présent article, nous nous intéressons principalement à l'hydrodynamique induite par la marée, en termes de variations spatiales des composantes de marée, ainsi que des courants, au sein du lagon.

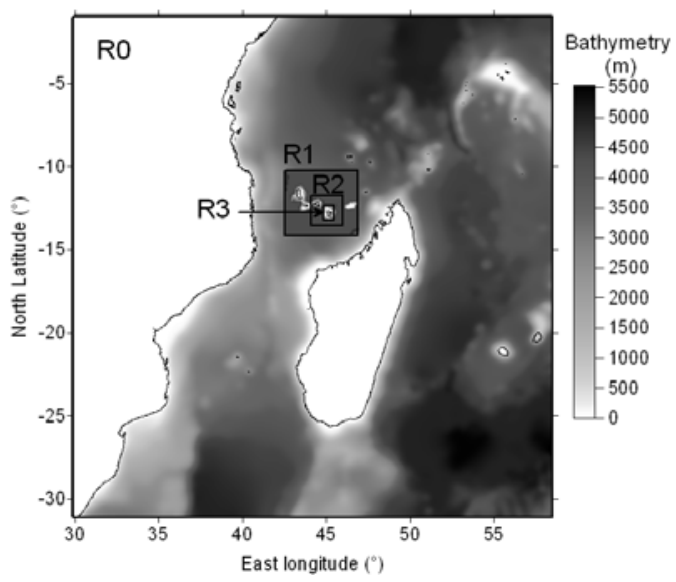

(a)

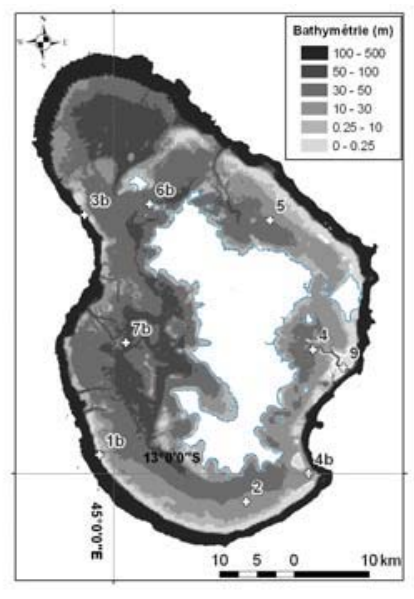

Figure 1 - (a) Emboitements pour la modélisation du lagon de Mayotte. Boites R0, R1, R2 et R3 (Mayotte), (b) bathymétrie du lagon et des marges externes. Localisation des capteurs déployés en novembre-décembre 2007. MNT issu de données bathymétriques SHOM et GEBCO.

\section{$2 \quad$ Mayotte : présentation}

La climatologie à Mayotte, située dans le Nord du canal du Mozambique, est régie par la Zone de Convergence Inter Tropicale (ZCIT). La ZCIT est une bande de basse pression d'environ $400 \mathrm{~km}$ de large, siège d'une confrontation entre les 
vents chauds et humides du nord et les vents plus froids et secs du sud.

Mayotte est située sur une branche du Courant Sud Equatorial (CSE), et au nord du canal du Mozambique, et donc à l'extrémité nord de la zone de tourbillons générés dans ce canal (SHOUTEN et al., 2003). SHOUTEN et al. (2003) montrent, par modélisation et observations satellites, des variations du niveau d'eau atteignant $5 \mathrm{~cm}$ autour de Mayotte, avec une période de 5 à 7 cycles par an (e.g. 50 à 70 jours), pour des courants, loin dans le canal du Mozambique, de l'ordre de quelques dizaines de $\mathrm{cm} / \mathrm{s}$. D'après (GOURBESVILLE et THOMASSIN, 2000), l'hydrodynamique du lagon ne serait pas influencée par les circulations générales de l'océan Indien. Le régime des marées, quant à lui est principalement semi-diurne, avec un marnage de $4 \mathrm{~m}$ en marée de vives-eaux.

SIEAM (2005) rappelle que sur le platier des récifs barrières, les courants rentrants sont induits par le déferlement de la houle du large et la marée, et que dans les lagons les courants sont principalement liés aux vents et à la marée, avec des courants entrant (resp. de vidange) de l'ordre $1 \mathrm{~m} / \mathrm{s}$ (resp. 1,5 m/s) dans les passes. Aussi, il est mis en évidence que le lagon de Mayotte n'est pas homogène sur le plan hydrologique (THOMASSIN et al., 1986).

\section{Observations in-situ}

\subsection{Campagne de mesure in-situ, données externes et contexte hydro- météorologique}

Afin de mieux connaitre les circulations, quatre courantomètres Aquadopp (capteurs 6b, 9, 1b, 7b) permettant d'acquérir des mesures en un point de la tranche d'eau, deux profileurs ADCP ( $3 b$ et $4 b$ ) et trois capteurs de pression OSSI ont été mouillés dans le lagon début novembre 2007, pendant un mois (Figure 1b). La durée de un mois est nécessaire pour pouvoir extraire les principales composantes de marée des mesures. Ces appareils ont été positionnés dans les passes principales du lagon, ainsi que dans les zones à fort fetch à l'intérieur du lagon. Les fréquences d'acquisition vont de $10 \mathrm{~Hz}$ pour les capteurs de pressions à $1 \mathrm{~Hz}$ pour les courantomètres. Ces mesures permettent donc de fournir des données de courants, et hauteurs d'eau pendant un mois, avec des jeux de données simultanées du 9/11 au 11/12. Le capteur 3b n'a pas fourni de données.

Afin de caractériser la météorologie à l'échelle des phénomènes des surcotes et ondes de tempête (phénomène qui est généré par les vents sur une emprise de plusieurs centaines à milliers de kilomètres, plus que par les vents locaux) pendant la campagne de mesure, les données de vent et pression de GFS ont été spatialement moyennées sur la zone Madagascar (emprise de la boite R0, Figure 1a). La Figure 2 montre que les vents étaient relativement modérés pendant la campagne (vent moyen de $2 \mathrm{~m} / \mathrm{s}$ ) et que la période du 23/11 au 26/11 est la période 
où les vents sont a priori négligeables. Pendant la campagne, en moyenne sur l'emprise de la boite R0, les vents venaient de Est-Sud-Est. En termes de pression, on note une pression moyenne de $1012 \mathrm{hPa}$, légèrement inférieure à la pression annuelle sur la même zone ( 1015 hPa d'après les données ECMWF).

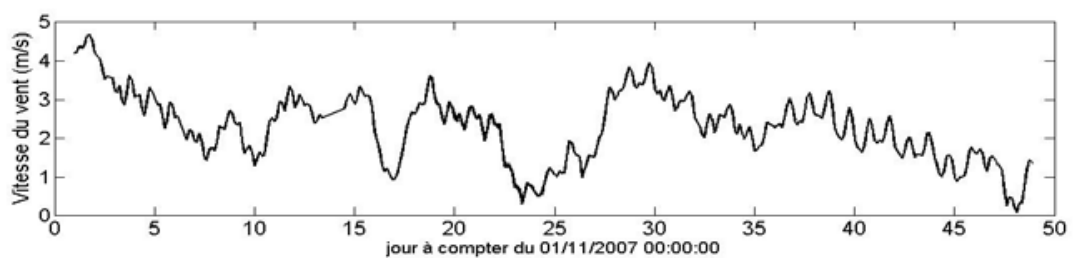

Figure 2 - Météorologie régionale (Emprise R0) à partir des sorties de modèle GFS : module du vent.

\subsection{Traitement des mesures hydrodynamiques}

Les mesures de pressions (donc hauteurs d'eau) et vitesses sont traitées pour dissocier signal de marée et signal résiduel (i.e. signal brut moins le signal de marée). L'estimation de la composante de marée est effectuée par analyse des composantes harmoniques (logiciel T_Tide, PAWLOWICZ et al., 2002). Notons que, dans un tel traitement appliqué à un mois de mesures, les périodes (des composantes de marée) les plus longues prises en compte sont de 20 jours. Les oscillations possibles, d'origine non-tidale, de 50 à 70 jours n'ont donc aucune influence sur le traitement de marée fait ici. Une fois les composantes de marée extraites (hauteur d'eau $h$, composantes de vitesse), le signal est recomposé en signal temporel. Afin de pouvoir comparer le modèle 2DH aux mesures, il s'agit d'estimer les vitesses $2 \mathrm{DH}$ aux points de mesures, à partir de mesures obtenues soit en un point de la tranche d'eau (capteurs dits « ponctuels » 6b, 9, 1b, 7b), soit en plusieurs points de la tranche d'eau (profileur 4b). Le traitement des données du profileur est fait en moyennant les mesures acquises sur toute la tranche d'eau. Le traitement des données des capteurs «ponctuels» nécessite de faire l'hypothèse que le profil vertical de vitesse suit une loi logarithmique. Dans cette loi, outre la mesure acquise à une cote $\mathrm{z}$ au-dessus du fond, il faut connaître la rugosité au fond, qui résulte de la rugosité de grain (sable fin estimé à $0,2 \mathrm{~mm}$, d'après les observations visuelles lors des mouillages) et de la rugosité de forme. Des tests de sensibilité en prenant ou non en compte la rugosité de forme due aux pinacles coralliens ont été effectués et montrent qu'ils peuvent être négligés dans ce traitement de la mesure.

\subsection{Niveaux d'eau}

Afin de vérifier la qualité de l'analyse T-tide, les niveaux d'eau bruts et les niveaux d'eau obtenus après analyse harmonique sur la durée de un mois sont 
comparées pendant une période de temps calme (22-23 novembre, Figure 3a). L'analyse T-Tide permet de reproduire le signal brut observé, et, avec un écart rms de $2 \mathrm{~cm}$. Sur cette même période de 2 jours, les capteurs donnent des écarts de 1 à $2,5 \mathrm{~cm}$ entre marée et niveau d'eau brut. L'analyse T-Tide peut donc être considérée comme satisfaisante.

Le tracé des composantes de marée en ces huit points (Figure 4) montre qu'il existe une variabilité spatiale des composantes. Aux positions 2,4,9,5,7b (Figure 1), les composantes restent relativement proches, tandis que les composantes en $1 \mathrm{~b}$ (passe Sud-Ouest), $4 \mathrm{~b}$ (passe Sud-Est) et $6 \mathrm{~b}$ (proche pointe Nord) montrent des différences de plusieurs dizaines de centimètres. Ainsi, par exemple entre les capteurs $1 \mathrm{~b}$ et $4 \mathrm{~b}$, l'onde M2 (resp. S2) diffère de $15 \mathrm{~cm}$ (resp. $12 \mathrm{~cm}$ ). On peut noter que jusqu'à présent les composantes de marée étaient considérées comme uniformes autour de Mayotte (GOURBESVILLE et THOMASSIN, 2000). Les mesures réalisées ici, sur un mois, permettent de quantifier les variations spatiales.
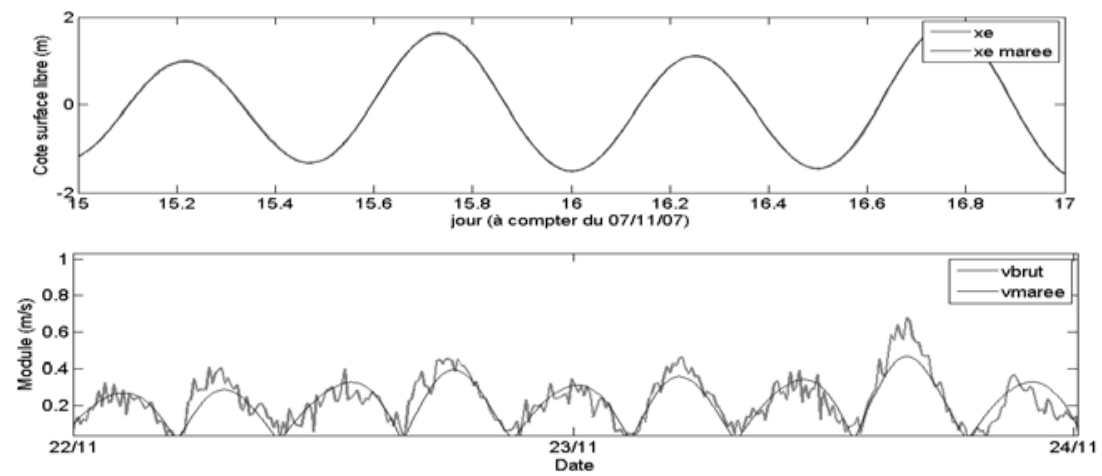

Figure 3 - Composantes brutes et composantes de marée (point $n^{\circ} 9$ ), pendant la période 22-24 novembre : (a) niveau d'eau (b) courant.

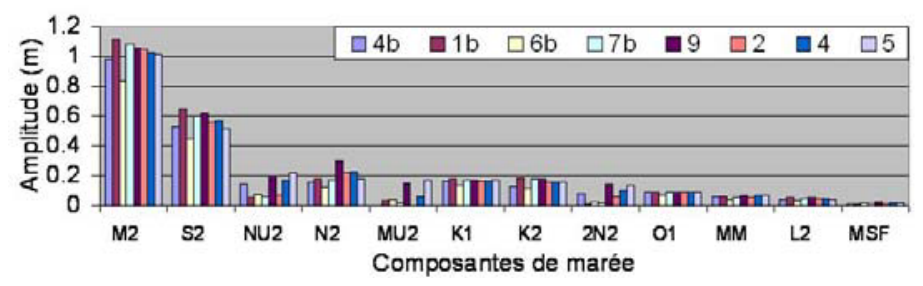

Figure 4 - Amplitude des composantes de marée obtenues par analyse T-Tide sur les huit capteurs.

\subsection{Courants}

Avant d'exposer les valeurs de courants $2 \mathrm{DH}$, obtenues après traitement des mesures, vérifions que l'hypothèse de profil logarithmique des vitesses est valide. Pour cela, nous utilisons les données du profileur $4 \mathrm{~b}$. Sur la majeure partie de la tranche d'eau (Figure 5), les courants vont quasiment tous dans la même direction, à l'exception de l'instant de renverse, tandis que le module des vitesses suit un 
profil relativement logarithmique. Ce type de profil conforte l'utilisation (1) d'un profil logarithmique pour estimer les courants $2 \mathrm{DH}$ à partir des mesures, et (2) d'une modélisation 2DH pour la courantologie du lagon de Mayotte.

Afin de vérifier la validité du traitement T-Tide sur les vitesses mesurées, le signal brut est comparé au signal de courant de marée obtenu par traitement $\mathrm{T}$ Tide (Figure 3b), pendant la période de temps calme du 22-24 novembre. On observe un bon accord. En fonction des capteurs, les écarts rms entre signal brut et signal tidal, sur cette période de deux jours, vont de 0,05 à $0,15 \mathrm{~m} / \mathrm{s}$. Le Tableau 1 donne quelques caractéristiques sur les mesures, après traitements. Il indique les courants maximaux 2DH totaux et tidaux, pendant la période 09/11/07-11/12/07. Selon les capteurs, la composante tidale est plus ou moins dominante dans le signal de courant. En termes de courants de marée $2 \mathrm{DH}$, on note une forte variabilité spatiale, avec des variations atteignant un facteur 6 (entre capteurs $7 \mathrm{~b}$ et 4b). Dans les passes (capteurs 1b, 9, 4b), les courants de marée 2DH atteignent des valeurs de l'ordre de $0,75 \mathrm{~m} / \mathrm{s}$, tandis que, à l'intérieur du lagon, dans les zones Nord (capteur 6b) et Ouest (capteur 7b), les courants sont plus faibles, de l'ordre de $0.15 \mathrm{~m} / \mathrm{s}$.
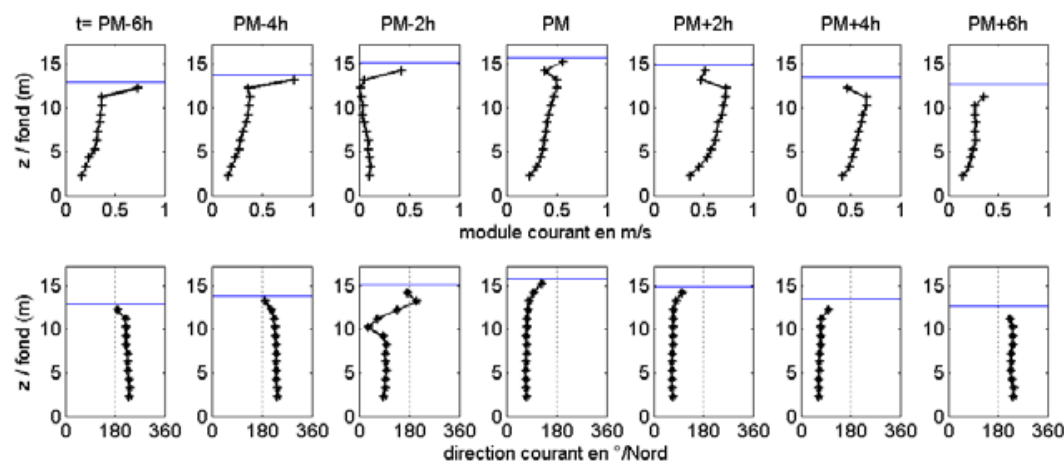

Figure 5 - Profils de vitesses par rapport à la Pleine Mer, le 23/11/2007. Capteur 4b. Les traits horizontaux indiquent le niveau de la surface libre.

Tableau 1-Grandeurs caractéristiques des mesures et résultats du modèle.

\begin{tabular}{|c|c|c|c|c|c|}
\hline \multirow{2}{*}{$\mathbf{N}^{\circ}$} & Type capteur & \multicolumn{2}{|c|}{ Mesures traitées } & \multicolumn{2}{c|}{ Modèle / mesures traitées } \\
\cline { 3 - 6 } & & $\begin{array}{c}\text { Umax total 2DH } \\
(\mathbf{m} / \mathbf{s})\end{array}$ & $\begin{array}{c}\text { Umax marée 2DH } \\
(\mathbf{m} / \mathbf{s})\end{array}$ & $\begin{array}{c}\text { rms - niveau de } \\
\text { marée (m) }\end{array}$ & $\begin{array}{c}\text { rms - courants de } \\
\text { marée (m/s) }\end{array}$ \\
\hline $\mathbf{7 b}$ & aquadopp & 0,41 & 0,15 & 0,08 & 0,05 \\
\hline $\mathbf{1 b}$ & aquadopp & 0,98 & 0,82 & 0,10 & 0,33 \\
\hline $\mathbf{9}$ & aquadopp & 0,86 & 0.67 & 0,07 & 0,27 \\
\hline $\mathbf{6 b}$ & aquadopp & 0,55 & 0,17 & 0,18 & 0,15 \\
\hline $\mathbf{4 b}$ & ADCP & 1,28 & 0,94 & 0,09 & 0,19 \\
\hline $\mathbf{2}$ & OSSI & & & 0,07 & \\
\hline $\mathbf{4}$ & OSSI & & & 0,08 & \\
\hline $\mathbf{5}$ & OSSI & & & 0,07 & \\
\hline
\end{tabular}




\section{$4 \quad$ Modélisation Hydrodynamique}

\subsection{Le modèle}

Afin d'avoir une connaissance plus générale des courants dans le lagon, un modèle hydrodynamique est mis en place avec le code MARS, développé par l'Ifremer, en version 2DH. Outre sa capacité à reproduire l'hydrodynamique en milieu lagonaire (DOUILLET, 1998), ce code a été choisi pour les facilités techniques suivantes qu'il apporte: mise en œuvre d'emboitements, prise en compte des forçages dus à la marée, ainsi que de conditions météorologiques instationnaires et non uniformes. La technique de modélisation emboitée est utilisée. Les conditions aux limites imposées sur les frontières de chaque boite sont les niveaux d'eau (marée, et les surcotes lorsque la météorologie est prise en compte). La boite d'emprise supérieure force la boite d'emprise inférieure.

Dans l'étude en cours, la météorologie est prise en compte dans les travaux de modélisation, mais dans le présent article, nous nous concentrons sur les résultats tidaux, e.g. sans prise en compte des conditions météorologiques. Alors que pour bien reproduire les surcotes il est nécessaires de modéliser l'écoulement sur des grands domaines (e.g. boite R0), pour modéliser l'hydrodynamique purement tidale, une emprise relativement locale des modèles est suffisante. Aussi, lors des calculs de la marée et des courants de marée, seules sont utilisées les boites R2 (Figure 1a), de taille de maille de $444 \mathrm{~m}$, et R3, plus petite, d'emprise égale au lagon, avec une taille de maille de $133 \mathrm{~m}$ (Figure 1a et 1b). Les données bathymétriques utilisées pour construire les Modèles Numériques de Terrain de chaque boite sont les données GEBCO, SHOM et des levés spécifiques effectués dans les passes (données DAF-Bambou Exploration). Les conditions aux limites sont issues des constantes de marée FES2004 (LYARD et al., 2006). En termes de frottement sur le fond, un coefficient non uniforme est imposé : $35 \mathrm{~m}^{1 / 3} \mathrm{~s}^{-1}$ par défaut et 20 sur les récifs coralliens.

\subsection{Niveaux d'eau}

Les mesures de hauteur d'eau traitées permettent d'estimer la qualité du modèle. Les écarts rms entre modèle et mesures traitées (uniquement composante tidale), calculées sur la période 09/11/07-11/12/07, vont de 0,07 à $0,10 \mathrm{~m}$ (Tableau 1). On peut noter les écarts rms au point $6 \mathrm{~b}$ qui sont de $0,18 \mathrm{~m}$. Ce capteur est mouillé tout près du rivage. Il est possible que le modèle, avec une taille de maille de 133 $\mathrm{m}$, ne permette pas de reproduire les données mesurées en ce point. Pour les autres capteurs, on note une relativement bonne adéquation modèle-mesure (Figure 6a).

\subsection{Courants}

Le Tableau 1 présente les écarts entre courants modélisés et mesurés. Ils sont 
d'autant plus faibles (en relatif) que les courants sont forts. Dans les passes larges (ex : passe Sud-Est), le modèle reproduit relativement bien les courants (Figure 6b) tandis qu'en dehors des passes, les courants sont sous-estimés, avec des écarts rms allant de 0,05 à $0,33 \mathrm{~m} / \mathrm{s}$ (Tableau 1). Les travaux de modélisation réalisés auparavant dans le lagon de Mayotte sous-estimaient aussi les courants observés, parfois jusqu'à un facteur 5, par exemple pour les travaux de SIEAM (2005). L'origine de cette sous-estimation n'a pas encore été clairement identifiée. Toutefois, notons que la sous-estimation est systématique à l'intérieur du lagon, et donc que le modèle permet quand même de dresser une image du lagon en termes de zonage hydrodynamique.
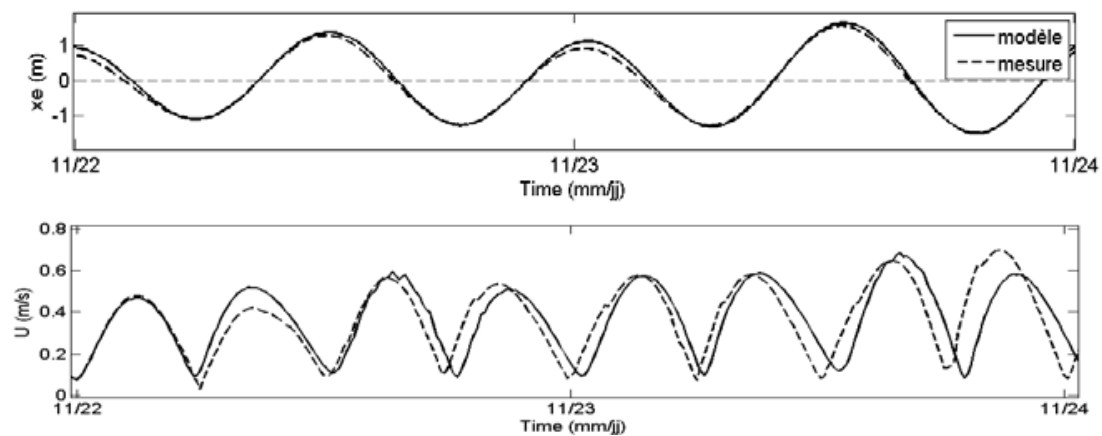

Figure 6-Modèle et données traitées par T-Tide, capteur 4b. (a) niveau d'eau et (b) courant.

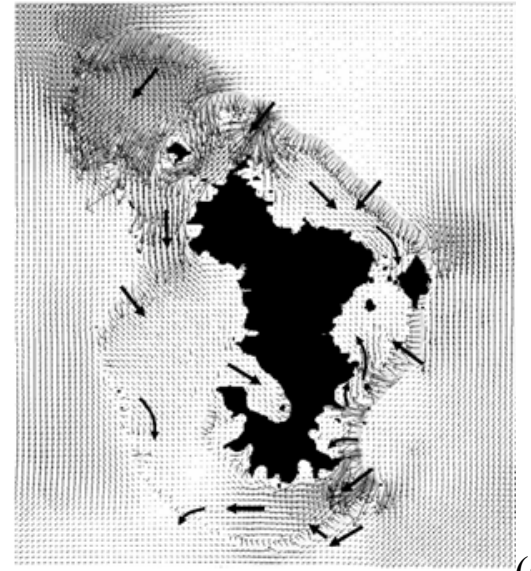

(a)

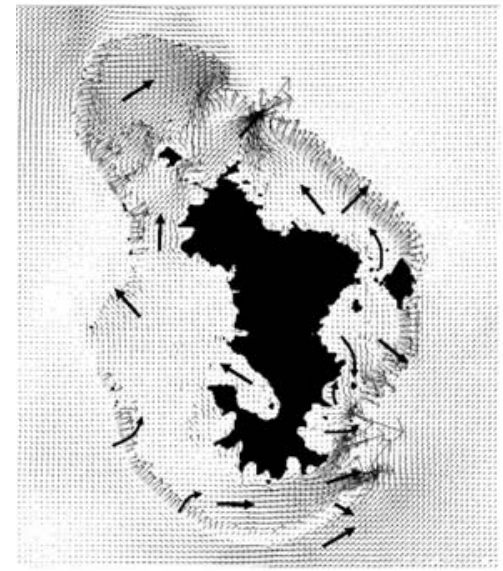

(b)

Figure 7 - Courants de marée le 21/11/07, au flot (a) et au jusant (b).Résultats du modèle.

La Figure 7 montre les directions des courants : au flot les courants entrent dans le lagon au niveau de toutes les passes et récifs ouest, nord, ainsi que le récif situé à l'ouest, tandis qu'au jusant, les courants rentrent par les passes et récifs du SudOuest et la barrière effondrée du Nord-Ouest. Ces résultats sont en accord avec les document existants réalisés à l'échelle du lagon (PORCHER et al., 2002), obtenus par compilation d'avis d'expert et d'études locales. 
Afin d'illustrer la variabilité spatiale des courants, la Figure 8 montre les variations spatiales des courants maximaux sur la période étudiée (09/11/07 au 11/12/07). On observe :

- Des zones à faible hydrodynamisme $(\mathrm{Umax}<0,2 \mathrm{~m} / \mathrm{s})$ située dans la zone Ouest du lagon (large de Tsingoni et Bouéni), ainsi qu'au Sud-Ouest de Petite Terre.

- Des zones à hydrodynamisme moyen $(0,2 \mathrm{~m} / \mathrm{s}<\operatorname{Umax}<0,8 \mathrm{~m} / \mathrm{s})$ situées au Sud et au Nord-Ouest et Nord-Est, dans les passes, ainsi qu'au large de la pointe de Saziley.

- Des zones à fort hydrodynamisme $(0,8 \mathrm{~m} / \mathrm{s}<$ Umax $)$ situées uniquement dans les passes.

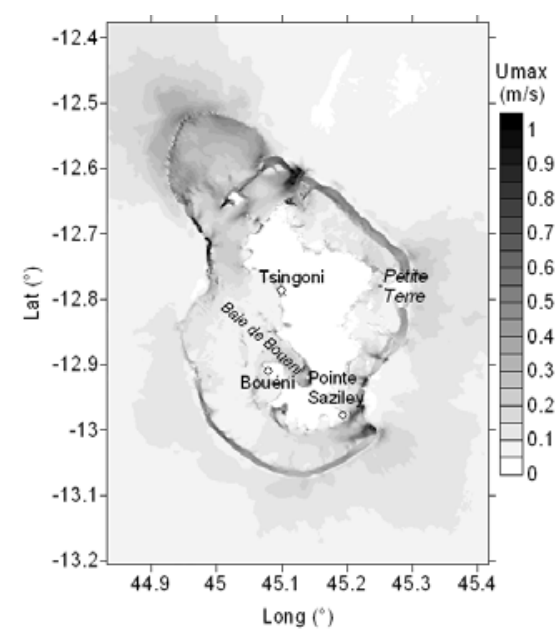

Figure 8 - Courants de marée maximaux (période : 09/11/07 au 11/12/07). Résultats du modèle.

\section{$5 \quad$ Conclusions}

Le présent article s'intéresse principalement à l'hydrodynamique tidale du lagon de Mayotte. Des mesures hydrodynamiques ont été réalisées en huit points dans le lagon et dans les passes. Ces mesures ont été analysées pour extraire les composantes tidales en termes de niveau d'eau et de courants. Ces analyses mettent en évidence des variations spatiales d'une part des composantes de marée, de l'ordre de $10 \mathrm{~cm}$ sur les composantes principales M2 et S2, d'autre part des courants de marée, variant d'un facteur 6 selon les capteurs, et atteignant $1 \mathrm{~m} / \mathrm{s}$ dans les passes.

Un modèle hydrodynamique $2 \mathrm{DH}$ a été mis en place. La comparaison avec les données traitées montre que le modèle estime correctement les intensités des courants dans les passes suffisamment larges, tandis qu'il les sous-estime au sein du lagon. Le modèle permet toutefois d'apporter une vision des courants de marée (directions et intensité) à l'échelle du lagon.

Les résultats présentés portent principalement sur l'hydrodynamique tidale. Afin d'avoir une connaissance plus complète des courants du lagon, il conviendra par la suite de prendre en compte les conditions météorologiques et l'action des vagues dans la modélisation des courants et niveaux d'eau. Les mesures réalisées pendant la campagne permettront d'estimer la qualité et les limites du modèle. Alors, l'hypothèse selon laquelle l'hydrodynamique de Mayotte dépend peu des grands courants océaniques pourra être vérifiée. 


\section{$\underline{\text { Remerciements }}$}

Ces travaux sont financés par la Direction de l'Agriculture et de la Forêt, la Collectivité Départementale de Mayotte et le BRGM. Les auteurs remercient l'IFREMER (en particulier Franck Dumas) de les avoir autorisés d'utiliser le code MARS pour ce projet, ainsi que Carlos Oliveros (BRGM) pour ses conseils.

\section{Références bibliographiques}

1 DOUILLET P. (1998) Tidal dynamics of south-west lagoon of New Caledonia: observations and 2D numerical modelling. Oceanologica Acta 21(1), 66-79.

2 GOURBESVILLE Ph., et THOMASSIN B.A. (2000), Coastal environment assessment procedure for sustainable wastewater management in tropical islands: the Mayotte example, Ocean and Coastal Management, 43, 997-1014.

3 IDIER D., PEDREROS R., OLIVEROS C., SOTTOLICHIO A., CHOPPIN L. et Bertin X., (2006), Contributions respectives des courants et de la houle dans la mobilité sédimentaire d'une plateforme interne estuarienne. Exemple : le seuil interinsulaire, au large du Pertuis d'Antioche, France. C.R. Geoscience, Vol. 338, 718-726.

4 LYARD F., F. LEFEVRE, T. LETELLIER and O. FRANCIS, (2006), Modelling the global ocean tides: modern insights from FES2004, Ocean Dynamics, DOI 10.1007/s10236-006-0086-x.

5 PAWLOWICZ R., BEARDSLEY B. and LENTZ S., (2002), Classical Tidal Harmonic Analysis Including Error Estimates in MATLAB using T_TIDE, Computers and Geosciences, 28, 929-937.

6 PORCHER P., SCHRIMM M., OBERLINKELS M., MORANCY R., NICOT S., GABRIE C., CHEMINE A., QUOD J.P., BIGOT L., ESBELIN C., THOMASSIN B.A., BLASCO F., FROMARD F., (2002) Volet 2 - Etat des lieux des milieux côtiers et récifo-lagonaires de Mayotte. Programme de protection et de mise en Valeur d'espaces Naturels d'intérêt écologique à Mayotte.

7 SCHOUTEN M., DE RUIJTERA W., JAN VAN LEEUWENA P., RIDDERINKHOF H. (2003) - Eddies and variability in the Mozambique Channel, Deep-Sea Research II 50, 1987-2003.

8 THOMASSIN, B.A., ARNOUX, A., COUDRAY, J., FROGET, C., GOUT, B., KOUYOUMONTZAKIS, G., MASSE, J.-P., REYRE, Y., REYS, J.-P., VACELET, E., (1989). La sédimentation actuelle dans le lagon de Mayotte (île volcanique à récif-barrière, SW Océan Indien) et son évolution récente en liaison avec les apports terrigènes. Bulletin de la Société Géologique de France 6, 1235- 1251.

9 Syndicat Intercommunal d'Eau et d'Assainissement de Mayotte (2005), Etudes préalables à la modification de la prise d'eau du dessalement et à la création d'un système d'assainissement à Petite Terre - Volume des Annexes Modélisation Maritime (SAFEGE). 